\title{
CoNi alloy nanoparticles for cancer theranostics: synthesis, physical characterization, in vitro and in vivo studies
}

\author{
Saman Sargazi ${ }^{1}$ Mohammad Reza Hajinezhad ${ }^{2} \cdot$ Abbas Rahdar $^{3} \cdot$ Mahwash Mukhtar $^{4}$. \\ Milad Karamzadeh-Jahromi ${ }^{5} \cdot$ Mohammad Almasi-Kashi $^{5,6} \cdot$ Sima Alikhanzadeh-Arani ${ }^{6,7} \cdot$ Mahmood Barani $^{8}$. \\ Francesco Baino ${ }^{9}$ (D)
}

Received: 7 July 2021 / Accepted: 8 September 2021 / Published online: 19 September 2021

(c) The Author(s) 2021

\begin{abstract}
Nanomaterials are attracting increasing interest in many biomedical fields, including the fight against cancer. In this context, we successfully synthesized CoNi alloy nanoparticles (NPs) by a simple polyol process. The magnetic characteristics of the products were measured by vibration sample magnometry, which revealed that the samples have soft ferromagnetic behavior. The microstructure and morphology were inspected by X-ray diffraction and scanning electron microscopy, respectively. Human cancer cells derived from the breast (MCF7) and oral cavity (C152) and normal cells derived from human umbilical vein endothelial cells (HUVECs) were treated with increasing concentrations of CoNi NPs, and their cytotoxic effect was measured via MTT and lactate dehydrogenase (LDH) leakage assays. We found that treatments by using 12.5 to $400 \mu \mathrm{g} / \mathrm{mL}$ of $\mathrm{Co} 0.5 \mathrm{Ni} 0.5, \mathrm{Co} 0.6 \mathrm{Ni} 0.4$, and $\mathrm{Co} 0.4 \mathrm{Ni} 0.6 \mathrm{NPs}$ were associated with significant concentration-dependent toxicity toward such cell lines and profoundly enhanced LDH leakage following $48 \mathrm{~h}$ of exposure $(P<0.05$ compared with untreated cells). Besides, a NP dose of $6.25 \mu \mathrm{g} / \mathrm{mL}$ did not affect the survival of HUVECs while leading to marked cell death in MCF7 and $\mathrm{C} 152$ cells. In vivo experiments in rats were done to investigate the biochemical and histopathological changes over three weeks, following intraperitoneal administration of $\mathrm{Co} 0.5 \mathrm{Ni} 0.5$, Co0.6Ni0.4, and Co0.4Ni0.6 NPs (100 mg/kg). As compared with the controls, the exposure to NPs caused significant elevations in aspartate aminotransferase, alanine aminotransferase, blood urea nitrogen, serum creatinine, serum catalase activity, serum superoxide dismutase, and liver malondialdehyde levels. Also, rats treated with Co0.6Ni0.4 NPs showed more severe histopathological changes of the liver and kidney. Our findings represent an essential step toward developing theranostic nanoplatforms for selective cancer treatment.
\end{abstract}

Keywords Nanomaterials $\cdot$ Metallic nanoparticles $\cdot$ In vivo $\cdot$ In vitro $\cdot$ Toxicity $\cdot$ Biomedical applications

\section{Introduction}

Presently, nanostructured alloys are getting attention in different fields because of their tailorable size and ability to retain the individual properties of each metal, which enable their wider use. The transition metal alloys have been widely explored for diagnostic and therapeutic purposes [1] because of their peculiar features, such as high saturation magnetization and good corrosion resistance [2]. Cobalt (Co) and nickel (Ni) are among the most commonly used transition

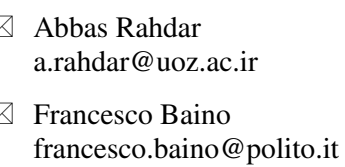

Extended author information available on the last page of the article metals for a range of advanced biomedical applications. Co is well known for its magnetic properties and is used as a contrast agent in magnetic resonance imaging (MRI) to investigate cancer and tumor cells, whereas $\mathrm{Ni}$ has potential use in the controlled magnetic hyperthermia of the malignancies [3]. CoNi alloys merge the fundamental characteristics of individual transition metals and, therefore, possess high magnetic properties, high thermal stability, and excellent wear resistance [4].

Nanotechnology has brought a revolution in the pharmaceutical and biomedical sciences. The nanoalloy systems have a high surface-to-volume ratio that can significantly affect the therapeutic or diagnostic functioning [5-11]. Currently, transition bimetallic alloys are gaining popularity due to their enhanced functionalities [12, 13]. Likewise, CoNi alloy nanoparticles (NPs) show suitable magnetic properties that can 
enhance the magnetic resonance signals to diagnose and target malignant cells. This enhanced MRI is possible because of the nature of CoNi NPs to overcome the superparamagnetic limit [14]. The existence of Co improves the conductivity of the alloy due to the charge hopping between $\mathrm{Co}$ and $\mathrm{Ni}$ cations [15]. CoNi nanoalloys have improved physical and chemical characteristics in contrast to elemental Co or Ni nanostructures. The synergy between these transition metals is linked to the enhanced stability with ultra-high-density magnetic imaging, microwave absorption and electromechanical strength [16]. Altogether, CoNi alloy NPs have anti-corrosive, electromagnetic, mechanical, and electrocatalytic functions that can be exploited in the electrical, mechanical and medical fields [17]. CoNi alloy NPs have consistently shown promising results in the hyperthermia treatment of malignant cells [18]. Besides, CoNi alloy NPs have been considered safe in biological applications according to different cell line studies. CoNi NPs have a potential application in reducing the electromagnetic interference problems that pose hazards to human health. This property is due to high microwave absorption intensity and wide bandwidth [19].

Several methods are employed for the synthesis of bimetallic alloy NPs [20, 21]. Different fabrication techniques utilized in the past to develop the CoNi alloy nanocarriers produced various shapes, morphology, and dimensions. The yielded nanocarriers can be categorized into zero-dimensional CoNi NPs, one-dimensional CoNi nanofibers, twodimensional $\mathrm{CoNi}$ nanofilms, and three-dimensional $\mathrm{CoNi}$ nanostructures [22-24]. Hence, the development of $\mathrm{CoNi}$ alloy NPs with desired attributes, productivity, and surface morphology is a challenging task. In the present work, we used the affordable and easy polyol process to synthesizing CoNi alloy NPs. In this method, the liquid polyols are used as a solvent, reducing agent, or complexing agent for the metallic precursors or cations. The precursor compounds are added to the polyols and heated to solubilize the metallic precursors. After cooling, the nano-crystalline powder is obtained. After producing the nanoalloys, we have explored the concentration-dependent cytotoxicity of the CoNi alloy NPs with varying ratios of $\mathrm{Co}$ and Ni. Our study also highlights the differences in the histopathological and biological studies of CoNi alloy NPs in a rat model. The developed CoNi alloy NPs were cytotoxic toward the malignant cells and show promise in medical theranostics for cancer treatment.

\section{Materials and methods}

\subsection{Materials}

Dulbecco's Modified Eagle's medium (DMEM), fetal bovine serum (FBS), and RPMI1640 were purchased from
Biochrom (Berlin, Germany). 3-(4, 5-Dimethylthiazol2-yl)-2, 5-diphenyltetrazolium bromide (MTT), trypan blue, phosphate-buffered saline (PBS), ethylene diamine tetra-acetic acid (EDTA), streptomycin, penicillin, and dimethyl sulfoxide (DMSO) were procured from Sigma-Aldrich (Steinheim am Albuch, Germany and Sigma-Aldrich, St Louis, MO). Plastic materials were provided by Jet-Biofill (Guangzhou, China).

\subsection{Preparation of NPs}

The simple and affordable polyol process was used for synthesizing the CoNi NPs, since this method has been previously proved to be effective for the preparation of metallic particles [25]. According to Fig. $1,40 \mathrm{~mL}$ of propylene glycol (PG) was heated to about $175{ }^{\circ} \mathrm{C}$. Then, the stoichiometric amounts of the precursors, $\mathrm{NiCl}_{2} \cdot 6 \mathrm{H}_{2} \mathrm{O}$ and $\mathrm{Co}\left(\mathrm{CH}_{3} \mathrm{COO}\right)_{2} \cdot 4 \mathrm{H}_{2} \mathrm{O}$, were homogeneously mixed and ground using mortar and pestle. The molar ratio of the metal salts was adjusted for three ratios, i.e., $\mathrm{Co}: \mathrm{Ni}=40: 60,50: 50$, and 60:40. The mixed precursors were added to the preheated PG. After about $5 \mathrm{~s}, \mathrm{NaOH}$ pellets were also added, and then the heater was turned off. The solution was then centrifuged twice at $6000 \mathrm{rpm}$ for $10 \mathrm{~min}$ with methanol and then with distilled water, and finally dried in an oven at $80{ }^{\circ} \mathrm{C}$ overnight.

\subsection{Characterizations}

The hydrodynamic size of the CoNi NPs in Milli-Q water was measured at $25{ }^{\circ} \mathrm{C}$ using dynamic light scattering (Malvern, Helix, UK). X-ray diffraction measurements were performed on the synthesized samples with a Rigaku D-max C III, X-ray diffractometer using $\mathrm{Ni}$-filtered $\mathrm{Cu} \mathrm{Ka} 1$ radiation

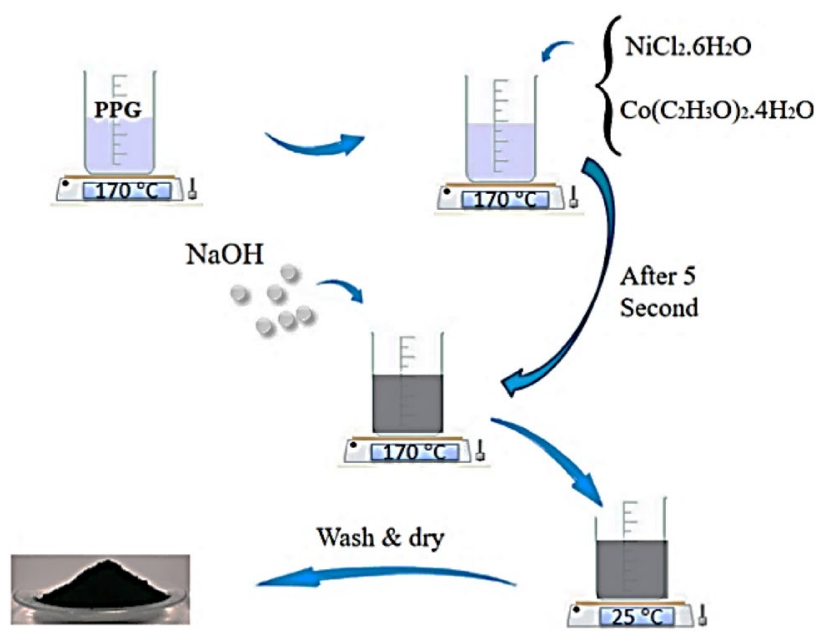

Fig. 1 Scheme illustrating the synthesis process of the CoNi nanoparticles 
$(\lambda \approx 1.5418 \AA$ ). Microscopic morphology of products was visualized by a LEO $1455 \mathrm{VP}$ scanning electron microscope (SEM). Magnetic measurements were carried out on a vibrating sample magnetometer (VSM) (Magnetic DaneshPajoh, I. R. Iran).

\subsection{Cells and culture condition}

Three cell lines were used in the current study. Michigan Cancer Foundation-7 (MCF-7) human breast cancer cells and $\mathrm{C} 152$ human mouth carcinoma were chosen as appropriate in vitro models for solid cancers. Therefore, these cell lines can be cultivated easily and are well-suited for cytotoxic evaluations on model cancerous cells. Human umbilical vein endothelial cells (HUVECs) were selected as a widely studied non-malignant cell line for cytotoxic evaluation on healthy cells. Cell lines were obtained from the National Cell Bank of Iran (Tehran, Iran) and tested negative for mycoplasma contamination. Cells were routinely cultured in RPMI1640 (MCF7) or DMEM (HUVEC, C152) supplemented with $10 \% \mathrm{FBS}$ and $100 \mathrm{U} / \mathrm{mL}$ penicillin-streptomycin under standard cell culture conditions (humidified atmosphere, $5 \% \mathrm{CO}_{2}, 37^{\circ} \mathrm{C}$ ). After reaching sub-confluency, the monolayer was washed with PBS and harvested using EDTA-Trypsin $0.25 \%$. Then, cells were resuspended in the culture medium and sub-cultured into $75 \mathrm{~cm}^{2}$ flasks and/or 96-well microplates for toxicity evaluations.

\subsection{In vitro cytotoxicity evaluation}

Cytotoxic effects of the synthesized NPs were assessed via MTT colorimetric assay. Cells were seeded at a density of $5 \times 10^{3}$ cells/well in a 96-well microplate and allowed to proliferate for $24 \mathrm{~h}$ before treatment. Untreated cells were considered as controls. Then, cells were exposed to increasing concentrations $(0,6.25,12.5,25,50,100,200$, and $400 \mu \mathrm{g} /$ $\mathrm{mL}$ ) of $\mathrm{Co} 0.5 \mathrm{Ni} 0.5, \mathrm{Co} 0.6 \mathrm{Ni} 0.4$, and $\mathrm{Co} 0.4 \mathrm{Ni0} .6 \mathrm{NPs}$, and incubated at aforementioned culturing conditions. After $48 \mathrm{~h}$ incubation, the culture medium was replaced with MTT dye $(5 \mathrm{mg} / \mathrm{mL})$ and kept in an incubator for another $3 \mathrm{~h}$. Finally, cell supernatant was discarded, and DMSO was added into each microwell to dissolve formazan crystals completely. The absorbance was read at $570 \mathrm{~nm}$ using a SpectraMax multi-plate reader (Molecular Devices, Sunnyvale, CA, USA). The percentage of viable cells was calculated by dividing the absorbance measured for treated cells by the absorbance measured for control cells $\times 100$. The GraphPad Prism 7.0Aa (San Diego, CA) software was used to calculate the half-maximal inhibitory concentrations (IC50s).

According to the manufacturer's protocol, the release of lactate dehydrogenase (LDH) was measured using a LDH assay kit (Cayman Chemical Co., Ann Arbor, MI). The LDH leakage (\% of positive control) was calculated using the following formula:

$(O D$ test $-O D$ blank $) /(O D$ positive $-O D$ blank $)$

where OD test is the absorbance of control cells, OD positive is the absorbance of the positive control cells or cells treated with increasing concentration of $\mathrm{Co} 0.5 \mathrm{Ni} 0.5$, Co0.6Ni0.4, and Co0.4Ni0.6 NPs for $48 \mathrm{~h}$, and OD blank represents the absorbance of the wells without cells. The absorption was read at $490 \mathrm{~nm}$ using a microplate reader (Spectra Max Gemini ${ }^{\circledR}$, Molecular Devices Cooperation, Sunnyvale, CA, USA).

\subsection{Animal treatments and experimental design}

Forty male Wistar rats were used in this study. Animals were obtained from the laboratory animal breeding colony of the faculty of veterinary medicine, University of Zabol, Zabol, Iran. Animals were kept in a well-ventilated room at normal temperature $\left(23-25{ }^{\circ} \mathrm{C}\right), 40 \%$ humidity and $12 \mathrm{~h}$ light-12 $\mathrm{h}$ dark cycle, and excellent ventilation. The experimental procedure was performed according to the guidelines of care and the use of laboratory rodents NIH publication no. 85-23 and was approved by the Ethical Committee of Zahedan University. Animals were randomly divided into four groups. Rats of the control group received intraperitoneal injections of saline. Animals in the other three groups received intraperitoneal administration of $100 \mathrm{mg}$ of Co0.5Ni0.5, Co0.6Ni0.4, and Co0.4Ni0.6 NPs per kilogram body-weight of rats. At the end of the study, blood samples were obtained by the retro-orbital sinus puncture. Samples were centrifuged (5000 rpm for fifteen min), and collected serum samples were kept at $-20{ }^{\circ} \mathrm{C}$ until analysis.

\subsection{Serum biochemical parameters}

The serum levels of blood urea nitrogen (BUN) and creatinine were measured according to Pars Azmoon reagent kits instructions (Pars Azmoon. Co., Tehran, Iran). All biochemical analyses were performed using the Selectra Pro M autoanalyzer (Vital Scientific, Netherlands). A colorimetric assay was used to determine serum aspartate aminotransferase (AST) and alanine aminotransferase (ALT) levels. Serum AST and ALT levels were measured using Pars Azmoon reagent kits (Pars Azmoon, Tehran, Iran). Serum and hepatic activities of catalase enzyme - an antioxidant enzyme-were determined using the Goth method [26].

Serum superoxide dismutase activity in serum samples was measured using the method described by Sun et al., with some modifications [27]. Total serum reduced glutathione (GSH) level was determined according to the protocol of Ellman et al. [28] and modified by Jollow et al. [29]. Liver 
malondialdehyde (MDA) contents were determined using the method described by Ohkawa et al. [30].

\subsection{Histopathological examinations}

After three months of oral administration, rats were euthanized by $1.5 \%$ pentobarbital sodium $(200 \mathrm{mg} / \mathrm{kg})$ followed by cervical dislocation. Liver and kidney specimens were sliced and preserved in 10\% neutral buffered formalin for two days to ensure complete tissue fixation. After paraffin embedding and cutting on a rotary microtome, paraffin blocks were cut into 5- $\mu \mathrm{m}$ microslides. The histopathological sections were stained with hematoxylin-eosin and examined under a light microscope (Tokyo, Olympus, Japan). The hepatic and renal lesions were graded from zero (normal histology) to three (severe pathological lesions) for semiquantitative analysis.

\subsection{Statistical analysis}

Data analysis was done using SPSS statistics software (SPSS Inc, version 23.0), and results were expressed as mean \pm SD from at least three independent experiments. Significant differences between the adjusted control and test condition were assessed using one-way analysis of variance (ANOVA), followed by post hoc analysis. Differences were considered statistically significant if $P$-value $<0.05$.

\section{Results}

\subsection{Characterization of NPs}

Figure 2 shows the typical XRD pattern of the synthesized Co0.4Ni0.6. The presence of three peaks at $2 \theta=44.47$, 51.74 , and $76.19^{\circ}$ corresponded to the face-centered cubic CoNi crystal structure with reference to JCPDS card No. 89-4307. The crystalline size was calculated to be $32 \mathrm{~nm}$ by the Debye-Scherrer equation.

The magnetic characteristics of the synthesized samples are represented in Fig. 3. It can be seen that the samples have the typical behavior of soft ferromagnetic materials. By increasing the amount of $\mathrm{Co}$, the magnetization increased too. The maximum magnetization (about $96 \mathrm{emu} / \mathrm{g}$ ) belonged to $\mathrm{Co} 0.6 \mathrm{Ni} 0.4 \mathrm{NPs}$. This sample also had a higher coercive field and squareness, of about 140 Oe and 0.12, respectively, compared to the other samples.

Due to the magnetic interaction, the synthesized NPs aggregated in the form of sub-microspheres observed in the SEM images of the prepared samples (Fig. 4). Their tendency to form clusters is consistent with previous observations on magnetic metallic NPs [31]. The particle size distribution histogram was calculated using the Digimizer

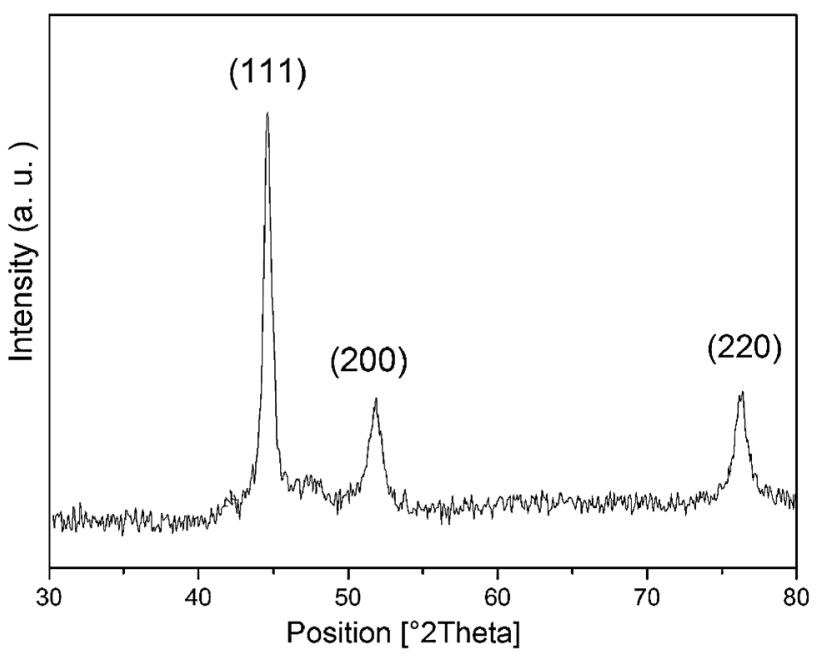

Fig. 2 The XRD pattern of the prepared Co0.4Ni0.6 NPs

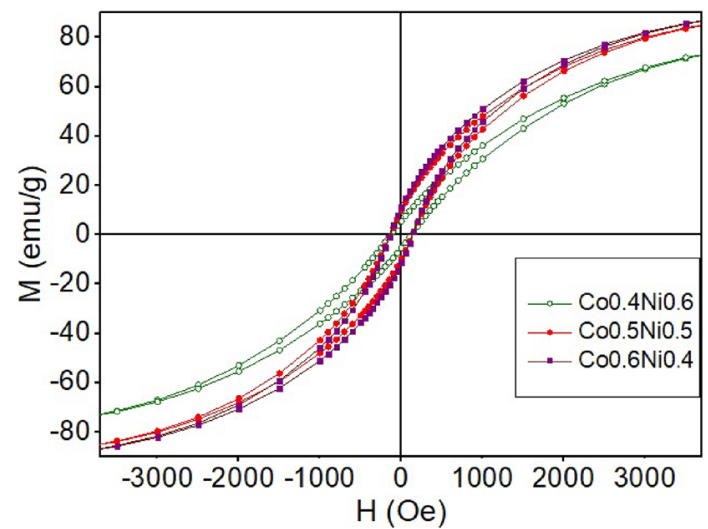

Fig. 3 Hysteresis loops of the prepared samples

software, as represented in Fig. 4. These results suggest that the diameter of the spheres decreased with the increasing content of $\mathrm{Ni}$.

The NPs were characterized by measuring the hydrodynamic size using DLS, but the shape and particle size of the NPs were studied using SEM. The hydrodynamic size of the Co0.5Ni0.5 NPs in Milli-Q water was found to be $297 \pm 5$ nm (Fig. 5).

\subsection{Determination of cell sensitivity to NPs}

We determined the reduction of MTT and leakage of LDH as reliable hallmarks of mitochondrial function and membrane integrity, respectively. Compared with control cells, Co0.5Ni0.5, Co0.6Ni0.4, and Co0.4Ni0.6 NPs showed concentration-dependent toxicity and a significant decrease in the number of alive MCF7, C152, and HUVEC cells (Fig. 6) $(P<0.05)$. The percentage of non-viable 
Fig. 4 The SEM images and size distribution of the prepared samples: a Co0.4Ni0.6, b $\mathrm{Co} 0.5 \mathrm{Ni} 0.5$, and $\mathbf{c} \mathrm{Co} 0.6 \mathrm{Ni} 0.4$
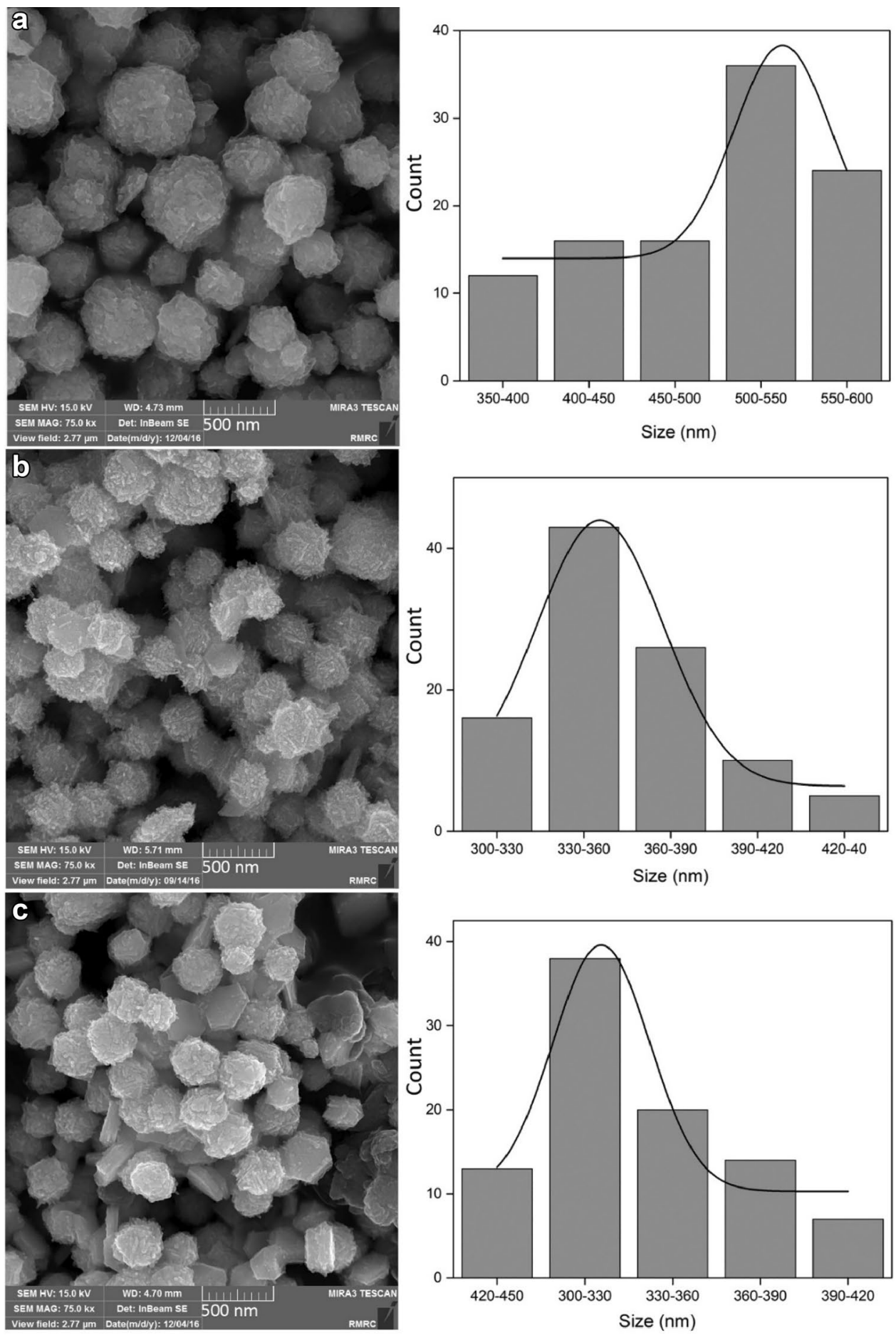

HUVEC cells following 48-h treatment with concentrations $6.25,12.5,25,50,100,200$, and $400 \mu \mathrm{g} / \mathrm{mL}$ of Co0.5Ni0.5 NPs was 12.66, 23.08, 25.90, 38.19, 67.33, 89.9 and $90.46 \%$, while these percentages were 5.90, 9.86, $27.88,64.15,78.34,88.37$ and $90.62 \%$ for $\mathrm{Co} 0.6 \mathrm{Ni} 0.4 \mathrm{NPs}$ and $6.81,12.36,40.97,74.50,87.34,90.23$ and $90.75 \%$ for Co0.4Ni0.6 NPs. IC50 concentration for treating HUVEC, C152, and MCF7 cells for $48 \mathrm{~h}$ with Co0.5Ni0.5 NPs was
$54.41,30.46$, and $28.04 \mu \mathrm{g} / \mathrm{mL}$, respectively. Interestingly, the three studied cell lines were more sensitive to Co0.6Ni0.4 and Co0.4Ni0.6 NPs.

IC50 concentration for treatment of cells with $\mathrm{Co} 0.6 \mathrm{Ni} 0.4$ and Co0.4Ni0.6 NPs in the given period was 42.63 and 31.41 for HUVEC cells, 22.17 and 18.85 for MCF7, and 18.14 and $14.62 \mu \mathrm{g} / \mathrm{mL}$ for $\mathrm{C} 152$ cells, respectively. Among the studied cell lines, non-malignant HUVEC cells were more resistant 


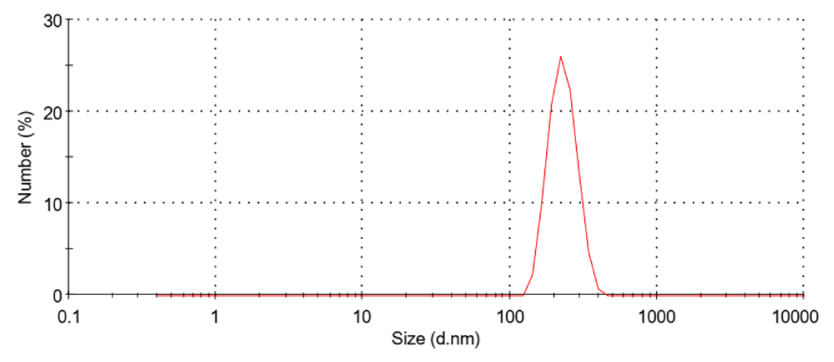

Fig. 5 The characterization of Co0.5Ni0.5 NPs by DLS

to the synthesized NPs, whereas the malignant $\mathrm{C} 152$ cells were more sensitive to CoNi NPs.

We assessed LDH leakage in HUVEC, C152, and MCF7 cells treated with increasing concentrations of CoNi NPs.
Compared with control cells, treatment of HUVEC cells with $6.25 \mu \mathrm{g} / \mathrm{mL}$ of $\mathrm{Co} 0.5 \mathrm{Ni} 0.5$, Co0.6Ni0.4, and Co0.4Ni0.6 NPs did not enhance LDH leakage $(\mathrm{P}>0.05)$. Nevertheless, compared to untreated cells, a significant increase in LDH leakage was noticed when HUVEC cells were exposed to a higher concentration of CoNi NPs $(\mathrm{P}<0.05)$ (Fig. 7). Exposure of MCF7 and $\mathrm{C} 152$ cells to $\mathrm{Co} 0.5 \mathrm{Ni} 0.5$, Co0.6Ni0.4, and $\mathrm{Co} 0.4 \mathrm{Ni} 0.6 \mathrm{NPs}$ resulted in a significant decrease in cell viability even at the lowest concentration $(\mathrm{P}<0.05$ compared with control cells) (Fig. 7).

\subsection{Biochemical results}

As shown in Table 1, control rats had normal serum BUN, serum creatinine, and serum liver enzyme levels. Serum AST and ALT levels of rats treated with Co0.5Ni0.5 were

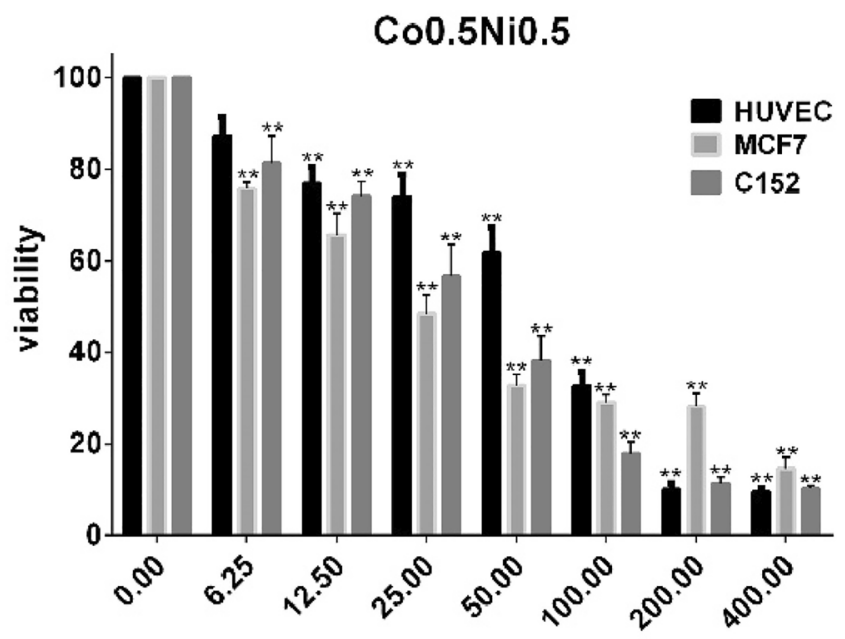

concentration $[\mu \mathrm{g} / \mathrm{mL}]$

\section{Co0.6Ni0.4}

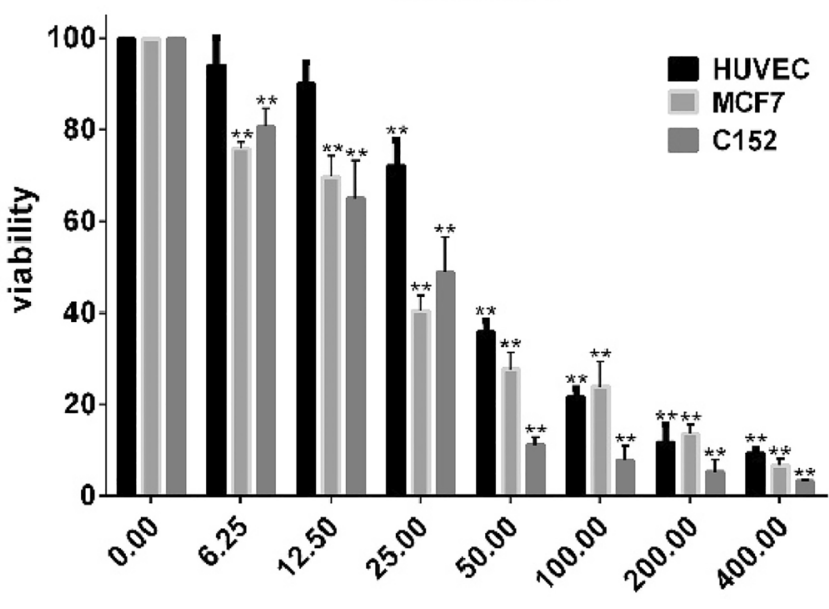

concentration $[\mu \mathrm{g} / \mathrm{mL}]$

Co0.4Ni0.6

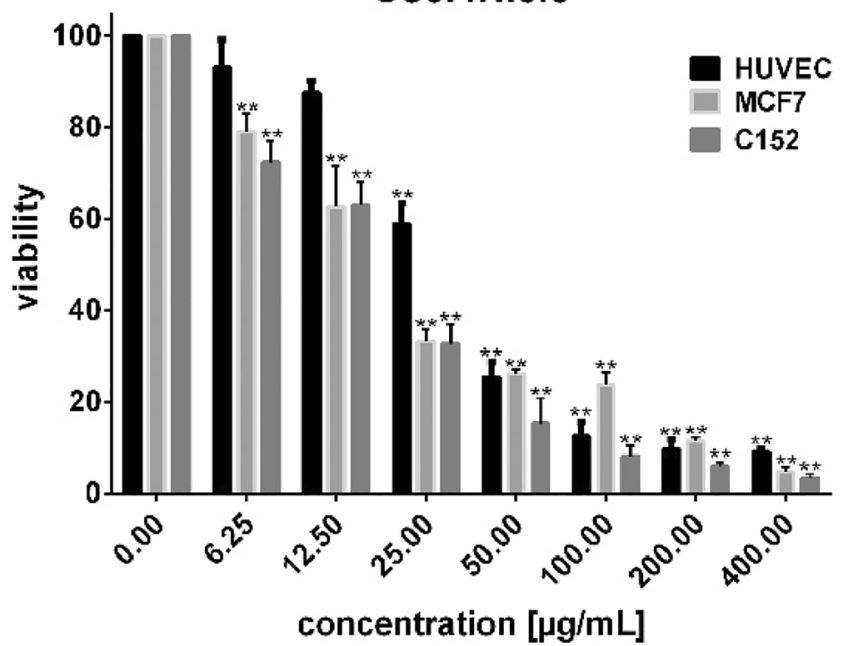

Fig. 6 Cytotoxicity assessment of Co0.5Ni0.5, Co0.6Ni0.4, and Co0.4Ni0.6 NPs on HUVEC, C152, and MCF7 cells NPs following 48 h of exposure $(* * P<0.05$ compared with control cells) 
Fig. 7 LDH leakage caused by $\mathrm{Co} 0.5 \mathrm{Ni} 0.5, \mathrm{Co} 0.6 \mathrm{Ni} 0.4$, and Co0.4Ni0.6 NPs after 48-h treatment
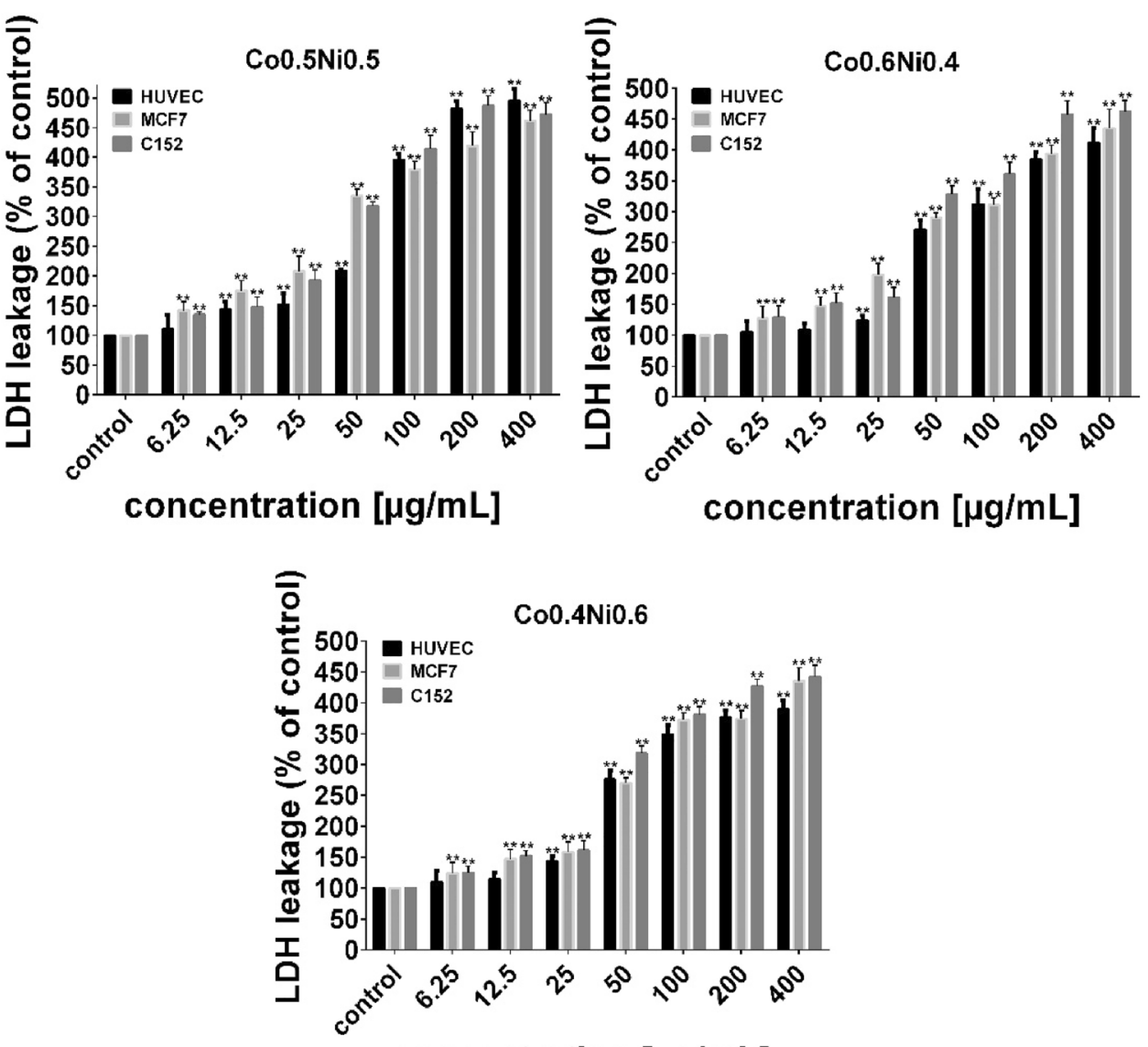

concentration $[\mu \mathrm{g} / \mathrm{mL}]$

significantly higher compared to the control rats $(P<0.001)$ (Table 1). There was also a significant increase in serum BUN and creatinine levels in rats treated with Co0.5Ni0.5 $(P<0.001)$. Rats of this group also had higher liver MDA levels when compared to the healthy control group $(P<0.001)$. Treatment with Co0.6Ni0.4 at the dose of $100 \mathrm{mg} / \mathrm{kg}$ also increased ALT and AST levels compared to the normal control rats. The statistical analysis showed a statistically significant difference in serum BUN and serum creatinine levels of rats treated with a $100 \mathrm{mg} / \mathrm{kg}$ dose of Co0.6Ni0.4 compared to healthy rats $(\mathrm{P}<0.001)$. There was also a statistically significant difference in serum AST, ALT, BUN, and creatinine levels of rats treated with Co0.4Ni0.6 $(100 \mathrm{mg} / \mathrm{kg})$ compared to the control rats $(\mathrm{P}<0.001)$. Liver
MDA levels also significantly increased in rats receiving the $100 \mathrm{mg} / \mathrm{kg}$ dose of $\mathrm{Co} 0.4 \mathrm{Ni} 0.6$ compared to the normal control rats, whereas liver MDA levels of rats receiving the $100 \mathrm{mg} / \mathrm{kg}$ of $\mathrm{Co} 0.4 \mathrm{NiO} .6$ were significantly higher than the normal control rats.

\subsection{Histopathological results}

Histopathological examination of kidney micrographs of control rats revealed normal renal glumerole and tubules, normal bowmen space, and healthy renal vascular system (Fig. 8a). The haematoxylin \& eosin staining of the liver of rats treated with $100 \mathrm{mg} / \mathrm{kg}$ dose of $\mathrm{Co} 0.5 \mathrm{Ni} 0.5$ showed intensive glomerular changes and glumerole sclerosis

Table 1 Effects of CoNi NPs on hematological parameters, biochemical parameters, and liver MDA content of different experimental groups

\begin{tabular}{|c|c|c|c|c|}
\hline Parameter & Control & Co0.5Ni0.5 (100 mg/kg) & Co0.6Ni0.4 (100 mg/kg) & Co0.4Ni0.6 (100 mg/kg) \\
\hline MDA (nmol/mg & $141.9 \pm 37.0$ & $211.0 \pm 13.6$ & $242.0^{* * * *} \pm 25.1$ & $222.2^{* * * *} \pm 48.7$ \\
\hline $\operatorname{AST}(\mathrm{U} / \mathrm{L})$ & $110.3 \pm 23.9$ & $145.6 \pm 32.4$ & $181.4^{*} \pm 19.7$ & $205.6^{* * *} \pm 13.7$ \\
\hline ALT (U/L) & $55.5 \pm 19.7$ & $118.5^{* * * *} \pm 30.0$ & $171.5^{* * *} \pm 80.0$ & $178.5^{* * * *} \pm 44.0$ \\
\hline BUN (mg/dL) & $12.1 \pm 2.0$ & $20.2^{* * * *} \pm 3.6$ & $22.0^{* * *} \pm 2.6$ & $25.1^{* * * *} \pm 3.7$ \\
\hline Creatinine (mg/dL) & $0.88 \pm 0.15$ & $1.26^{* * * *} \pm 0.30$ & $1.75^{* * * *} \pm 0.40$ & $1.70^{* * *} \pm 0.44$ \\
\hline
\end{tabular}


(Fig. 8b). Renal histopathological changes of rats receiving the $100 \mathrm{mg} / \mathrm{kg}$ dose of $\mathrm{Co} 0.6 \mathrm{Ni} 0.4$ showed more severe histological changes, including extensive parenchymal hemorrhage (Fig. 8c). The kidney section of rats treated with $100 \mathrm{mg} / \mathrm{kg}$ dose of Co0.4Ni0.6 showed extensive morphological changes of renal tubules (narrowing) and cytoplasmic vacuolation (Fig. 8d).

Histopathological section of the healthy rats' kidneys showed normal structure, normal hepatocytes, and wellarranged lobule sinusoids (Fig. 9a). Histopathological investigation of a liver micrograph of rats treated with $\mathrm{Co} 0.5 \mathrm{NiO} .5$ showed slight disarrangement of sinusoids (Fig. 9b). Liver histological micrograph of rats received $100 \mathrm{mg} / \mathrm{kg}$ dose of Co0.6Ni0.4 mg/kg, and arrow showed hepatocyte necrosis, vacuolation of hepatocytes, and nuclear pyknosis (Fig. 9c). Histopathological micrograph of liver of rats treated with $100 \mathrm{mg} / \mathrm{kg}$ dose of $\mathrm{Co} 0.4 \mathrm{NiO} .6$ showed hemorrhage and hepatocyte pyknosis (Fig. 9d).

\section{Discussion}

The combination of imaging and therapeutics provides excellent control over the efficacy of current cancer treatments [32]. Besides having adequate biocompatibility and
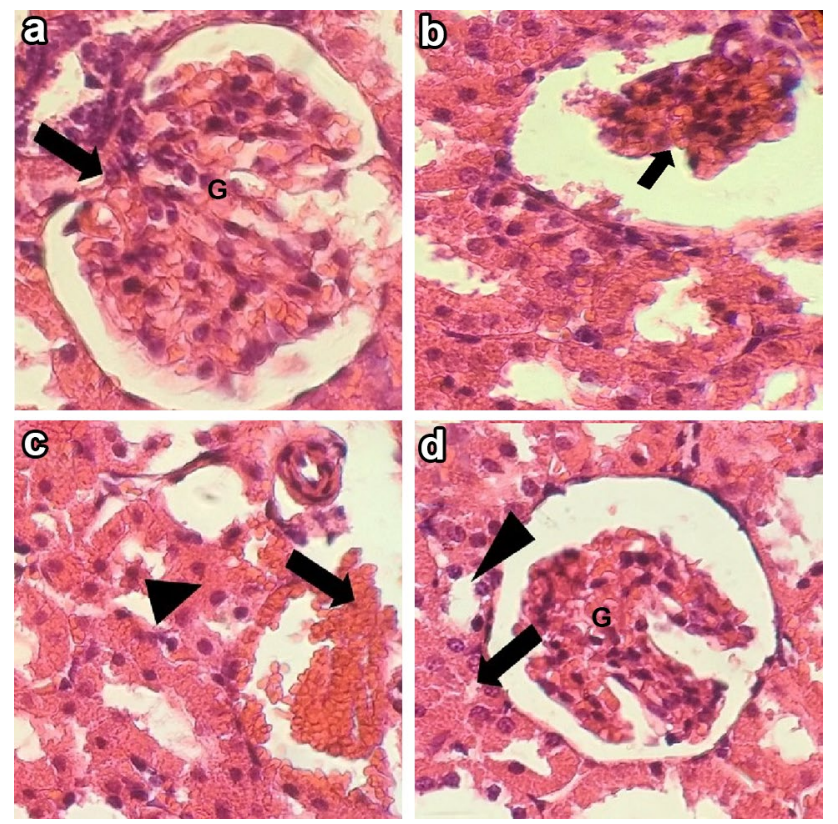

Fig. 8 a Histopathological section of a kidney of a control rat showing $(\mathrm{G})$ normal glomeruli; b Kidney sections of a rat treated with Co0.5Ni0.5 showing glumerole sclerosis (arrow).; c Kidney section of a rat received $100 \mathrm{mg} / \mathrm{kg}$ dose of Co0.6Ni0.4 mg/kg, arrow shows hemorrhage, arrowhead showing deformation of renal tubules.; d Kidney section of a rat treated with $100 \mathrm{mg} / \mathrm{kg}$ dose of Co0.4Ni0.6, morphological changes of renal tubules (arrowhead) and cytoplasmic vacuolation (arrow), H\&E staining $(\times 40)$
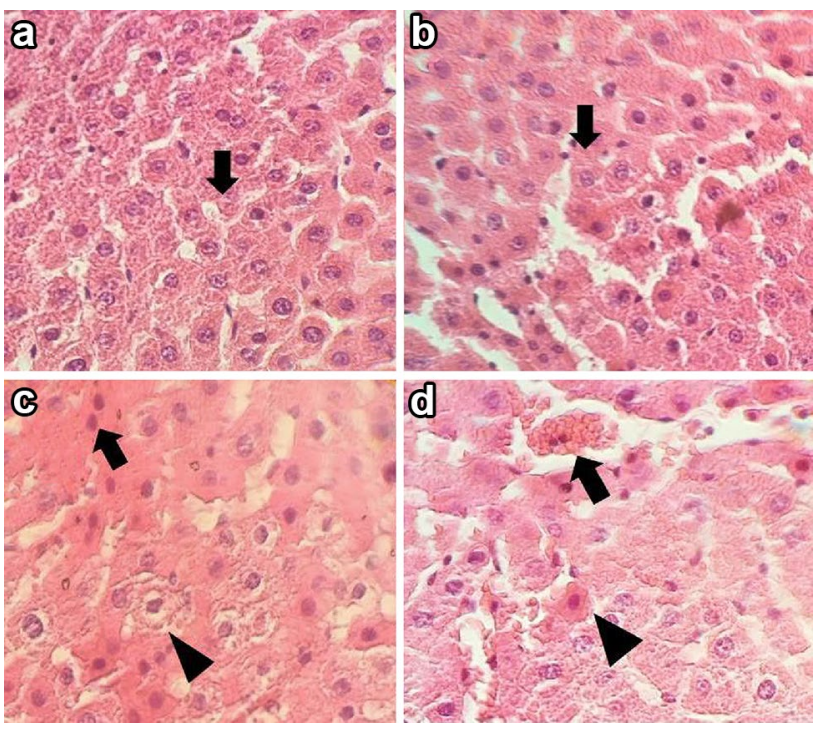

Fig. 9 a Histopathological section of liver of a control rat showing (G) normal hepatocytes and hepatic sinusoids; liver micrograph of a rat treated with $\mathrm{Co} 0.5 \mathrm{Ni0.5}$ showing sinusoidal disarrangement $(\mathrm{G})$; c Liver histological micrograph of a rat received $100 \mathrm{mg} / \mathrm{kg}$ dose of Co0.6Ni0.4 mg/kg, and arrow shows necrosis, arrowhead showing vacuolation of hepatocytes; d Histopathological micrograph of liver of a rat treated with $100 \mathrm{mg} / \mathrm{kg}$ dose of Co0.4Ni0.6, hemorrhage (arrow) and hepatocyte pyknosis (arrowhead), H\&E staining $(\times 40)$

small nanoscale size, magnetic NPs provide a unique platform for theranostic uses [33]. However, one of the most critical challenges in employing magnetic NPs is their safety in healthy cells [34].

In the current study, we aimed to prepare small-size CoNi NPs and investigate their biological activities using in vitro and in vivo models. Interestingly, we found that magnetic $\mathrm{Co} 0.5 \mathrm{Ni} 0.5$, Co0.6Ni0.4, and $\mathrm{Co} 0.4 \mathrm{Ni} 0.6 \mathrm{NPs}$ tended to agglomerate forming clusters with $\mathrm{Ni}$-dependent size, exhibited concentration-dependent toxicity, and disrupted the membrane integrity of normal and malignant cells to different extents. In this context, it can be concluded that the synthesized CoNi NPs exert beneficial cell-killing effects against malignant cells. In contrast, normal human cells were less affected during 48-h treatment. It might be a promising outcome, as we found a safe dose for the synthesized NPs to be employed in further biomedical applications without creating any harmful effects in humans.

A substantial limitation inherent to various traditional anti-tumor agents is their lack of tumor selectivity and inability to enter the core of tumors, leading to impaired treatment with decreased dose and low survival rate [35, 36]. It has been previously shown that small-size metallic NPs can passively accumulate in cancerous cells due to the enhanced permeation and retention (EPR) effect $[37,38]$. The EPR effect is the property by which specific sizes of molecules (i.e., NPs) accumulate more in tumor tissues than in normal 
tissues, therefore lowering the systematic toxicity of these molecules [38]. On the other hand, the accumulation of a high amount of some metallic NPs in cancerous cells/tissues increases cell death in these cells [39]. For instance, the accumulation of silver NPs causes cell death via the generation of reactive oxygen species and inducing DNA damage [40]. This might be the rationale for the higher cytotoxicity of CoNi NPs against cancer cells compared to normal cells.

Over the past few years, the toxicity of metal oxide NPs has aroused major concerns. Still, it is unclear whether their toxicity comes from the NPs themselves or the released ions [41]. Magaye et al. demonstrated that intravenous injection of Ni NPs $(50 \mathrm{~nm})$ through the dorsal penile vein of rats caused severe spleen, liver, and cardiac toxicity and lung inflammation [42]. Gornati and coworkers investigated the cytotoxicity of Co and Ni NPs on SKOV3 (ovarian cancer) and U87 (human primary glioblastoma) cells. Their findings revealed that Ni NPs released a much smaller amount of ions in the medium than Co NPs. Both NPs have shown cytotoxic effects in cancer cells. However, interestingly, the HSP70 gene encoding a heat shock protein was only upregulated in SKOV3 by both NPs. This suggests that metallic NPs alter the expression of genes involved in cellular stress response (i.e., HSP70, MT2A, SDHB1, and $M L L$ ) or apoptotic cell death (caspase3) on a case-by-case basis, and their growthinhibitory effects should be investigated in different in vitro models [43]. In a similar study, Ibrahim and colleagues synthesized superparamagnetic Ni NPs with spherical shapes and assessed their possible toxicity against non-cancerous fibroblastic cells and cancerous prostate cancer cells. In contrast with previous findings, they observed that these NPs exhibit no acute toxicity in the studied cell lines, proposing that the prepared magnetic Ni NPs are considerably biocompatible in vitro and might have potential biomedical applications [44].

Furthermore, metallic NPs might exert synergistic effects when combined with anticancer drugs [45-47]. Guo et al. showed that Ni NPs significantly enhance the permeability of cancerous SMMC-7721 hepatocellular carcinoma cells and increase the accumulation of quercetin in these cells [48]. In another study, Guo's research team examined the effects of Ni NPs on improving cellular uptake of daunorubicin, and found a synergistic interaction between these two agents on efficient growth inhibition of leukemia cancer cells [49]. In 2020, Zhang and colleagues developed a multifunctional platform for cancer therapy by synthesizing polyethylene glycol (PEG) modified cobalt carbide NPs via a hightemperature thermal decomposition method. The prepared NPs acted as a theranostic agent for imaging modalities and photothermal therapy and demonstrated excellent efficacy for cancer management both in vitro and in vivo [50]. Ahamed et al. investigated the effects of Ni-ferrite NPs on the growth of MCF7 breast cancer and HepG2 liver cancer cells and observed that MCF7 cells were slightly more sensitive to the prepared NPs than HepG2 cells. Moreover, the anti-apoptotic gene $b c l-2$ was down-regulated following treatment of cancer cells with Ni-ferrite NPs [51]. Magaye et al. suggested that metallic Ni NPs may exhibit higher anticancer activities than fine particles in JB6 cells [52]. Lei et al. suggested that optimizing Ni-based photoabsorbers using near-infrared light can be a promising strategy for cancer photothermal therapy [53]. Magnetic liposomes based on Ni-ferrite NPs have also introduced as innovative nanocarriers for cancer therapy [37]. These observations were in agreement with the findings of the current study, suggesting that small-sized metallic NPs with unique biochemical activity can selectively target cancer cells and, thus, provide an opportunity for targeted cancer therapy.

Ansari et al. showed that Co NPs induced mild growth inhibitory effects toward A2780cp cisplatin-resistant ovarian cancer cells while exhibited no toxicity against normal human cells [54]. Based on MTT results, we observed that treatment of normal human cells with $6.25 \mu \mathrm{g} / \mathrm{mL}$ of Co0.5Ni0.5, Co0.6Ni0.4, and Co0.4Ni0.6 NPs did not induce any significant toxicity. Still, Co0.4Ni0.6 seemed to be more toxic than the other two CoNi NPs due to lower IC50s in all three cell lines. Previously, Peymani-Motlagh and colleagues assessed the growth-inhibitory effects of $\mathrm{Co}_{0.5} \mathrm{Ni}_{0.5} \mathrm{Pr}_{0.1} \mathrm{Fe}_{1.9} \mathrm{O}_{4}$ magnetic NPs on $\mathrm{A} 549$ human lung cancer cells and reported no significant cytotoxicity at concentrations of up to $500 \mu \mathrm{g} / \mathrm{mL}$ following treatment for $24 \mathrm{~h}$ [55]. Unlike their results, we found concentration-dependent toxicity for the synthesized NPs toward cancer cells. Still, the synthesized CoNi NPs exerted negligible or no noncytotoxic effects at $6.25 \mu \mathrm{g} / \mathrm{mL}$.

As regards in vivo tests, the group treated with Co0.6Ni0.4 had more severe histopathological and biochemical changes. In the former studies, both $\mathrm{Co}$ and $\mathrm{Ni}$ NPs showed nephrotoxicity, cardiotoxicity, and hepatotoxicity in laboratory mice and rats [56, 57]. Our results showed nephrotoxicity and hepatotoxicity of $\mathrm{Co} 0.5 \mathrm{Ni} 0.5$, $\mathrm{Co} 0.6 \mathrm{Ni0} .4$, and Co0.4Ni0.6 NPs. There was no significant difference in serum biochemical parameters of rats treated with different Ni-and Co-based NPs; however, histopathological results showed that $\mathrm{Co} 0.6 \mathrm{Ni} 0.4$ could induce more undesirable effects. Earlier review articles demonstrated that Co NPs have more severe side effects compared to Ni NPs. Previous studies have also shown that NPs consisting of two different elements could have different biological effects than each NP alone. It is not fully understood how Ni-and Co-based NPs could induce histopathological changes in the liver and kidney. Increased lipid peroxidation, decreased antioxidant enzyme function, and atriovascular changes may play significant roles in the hepatotoxicity and nephrotoxicity of Ni-and Co-based NPs. Also, the use of machine learning and molecular dynamic simulation can 
help for better understand and function of NPs [58-61]. Altogether, we have found that the prepared CoNi NPs could induce in vitro cytotoxic effects. However, the safety profile of these metallic NPs against normal human cells should be further studied.

\section{Conclusion}

Through performing in vitro cytotoxicity assessments, we observed that the $\mathrm{Co} 0.5 \mathrm{Ni} 0.5$, $\mathrm{Co} 0.6 \mathrm{Ni} 0.4$, and $\mathrm{Co} 0.4 \mathrm{Ni} 0.6$ NPs with the dose of $6.25 \mu \mathrm{g} / \mathrm{mL}$ did not induce undesirable effects against normal human cells, while eliciting marked cell death in cancerous cells. This can be due to the EPR effects explaining that small-sized CoNi NPs accumulate more selectively in cancerous cells than in normal cells. Similarly, all the three forms of CoNi NPs showed toxic effects on rats, with Co0.6Ni0.4 NPs showing the highest toxic effects. Our findings represent an essential step toward developing theranostic nanoplatforms for cancer treatment. Still, further investigations are required to elucidate the toxicity/benefit ratio associated with the prepared NPs for selective potential applications in clinics.

Funding Open access funding provided by Politecnico di Torino within the CRUI-CARE Agreement. This study received funding from Zahedan University of Medical Sciences (Project. 10267).

Availability of data and material Data are included within this article.

\section{Declarations}

Conflicts of interest The authors declare no conflict of interest.

Ethics approval The in vitro part of the study protocol was approved by Zahedan University of Medical Sciences (Ethical code: IR.ZAUMS. REC.1399.517).

Open Access This article is licensed under a Creative Commons Attribution 4.0 International License, which permits use, sharing, adaptation, distribution and reproduction in any medium or format, as long as you give appropriate credit to the original author(s) and the source, provide a link to the Creative Commons licence, and indicate if changes were made. The images or other third party material in this article are included in the article's Creative Commons licence, unless indicated otherwise in a credit line to the material. If material is not included in the article's Creative Commons licence and your intended use is not permitted by statutory regulation or exceeds the permitted use, you will need to obtain permission directly from the copyright holder. To view a copy of this licence, visit http://creativecommons.org/licenses/by/4.0/.

\section{References}

1. L.P. Zhu, H.M. Xiao, S.Y. Fu, Surfactant-Assisted Synthesis and Characterization of Novel Chain-like CoNi Alloy Assemblies (Wiley, New York, 2007)

2. S. Bai et al., In situ growth of $\mathrm{Ni} x$ Co100-x nanoparticles on reduced graphene oxide nanosheets and their magnetic and catalytic properties. ACS Appl. Mater. Interfaces. 4(5), 2378-2386 (2012)

3. R. Magaye et al., Genotoxicity and carcinogenicity of cobalt-, nickel-and copper-based nanoparticles. Exp. Ther. Med. 4(4), 551-561 (2012)

4. M.J. Hu, B. Lin, S.H. Yu, Magnetic field-induced solvothermal synthesis of one-dimensional assemblies of Ni-Co alloy microstructures. Nano Res. 1(4), 303-313 (2008)

5. A. Badoei-Dalfard et al., Fabrication of an efficient and sensitive colorimetric biosensor based on Uricase/Th-MOF for uric acid sensing in biological samples. Biosens. Bioelectr. 141, 111420 (2019)

6. M. Ehrampoush et al., Removal of methylene blue (MB) dye from textile synthetic wastewater using $\mathrm{TiO} 2 / \mathrm{UV}-\mathrm{C}$ photocatalytic process. Aust. J. Basic Appl. Sci. 4(9), 4279-4285 (2010)

7. M. Malakotian et al., Protocol encompassing ultrasound/Fe $3 \mathrm{O} 4$ nanoparticles/persulfate for the removal of tetracycline antibiotics from aqueous environments. Clean Technol. Environ. Policy 21(8), 1665-1674 (2019)

8. A. Miri et al., Cerium oxide nanoparticles: green synthesis using Banana peel, cytotoxic effect, UV protection and their photocatalytic activity. Bioprocess Biosyst. Eng., 2021: 1-9.

9. A. Miri, M. Sarani, M. Khatami, Nickel-doped cerium oxide nanoparticles: biosynthesis, cytotoxicity and UV protection studies. RSC Adv. 10(7), 3967-3977 (2020)

10. M. Barani et al., Nanotechnology in ovarian cancer: Diagnosis and treatment. Life Sciences, 2020: 118914.

11. M. Barani et al., Progress in the application of nanoparticles and graphene as drug carriers and on the diagnosis of brain infections. Molecules 26(1), 186 (2021)

12. X. Deng et al., Design and fabrication of a novel dual-frequency confocal ultrasound transducer for microvessels super-harmonic imaging. IEEE Trans. Ultrason. Ferroelectr. Freq. Control 68(4), 1272-1277 (2020)

13. X. Du et al., Lead halide perovskite for efficient optoacoustic conversion and application toward high-resolution ultrasound imaging. Nat. Commun. 12(1), 1-9 (2021)

14. H. Li et al., Hollow CoNi alloy submicrospheres consisting of CoNi nanoplatelets: facile synthesis and magnetic properties. Mater. Lett. 67(1), 346-348 (2012)

15. Q. Sheng, D. Liu, J. Zheng, NiCo alloy nanoparticles anchored on polypyrrole/reduced graphene oxide nanocomposites for nonenzymatic glucose sensing. New J. Chem. 40(8), 6658-6665 (2016)

16. K. Rajar, E. Alveroglu, Hydrogel covered bimetallic Co: Ni magnetic nano alloy for protein adsorption in biomedical application. J. Mol. Struct. 1146, 592-599 (2017)

17. Parajuli, S., et al., Diameter dependent structural and magnetic properties of CoNi alloy nanotubes. Journal of Magnetism and Magnetic Materials, 2020. 500: p. 166264.

18. S.N. Kale, et al., Characterization of biocompatible NiCo2O 4 nanoparticles for applications in hyperthermia and drug delivery. Nanomedicine: Nanotechnology, Biol. Med., 2012. 8(4): 452-459.

19. $\mathrm{Ni}, \mathrm{C}$., et al., Microwave absorption properties of microporous CoNi@(NiO-CoO) nanoparticles through dealloying. Journal of Magnetism and Magnetic Materials, 2020. 503: p. 166631.

20. H. Chen, C. Xu, Surfactant-assisted hydrothermal synthesis of 3D urchin-like cobalt-nickel microstructures. Mater. Lett. 162, 13-16 (2016) 
21. M. Cheng et al., Solvothermal synthesis of NiCo alloy icosahedral nanocrystals. Inorg. Chem. 51(3), 1495-1500 (2012)

22. N.A. Barakat et al., CoNi bimetallic nanofibers by electrospinning: nickel-based soft magnetic material with improved magnetic properties. J. Phys. Chem. C 114(37), 15589-15593 (2010)

23. O. Ergeneman et al., Morphology, structure and magnetic properties of cobalt-nickel films obtained from acidic electrolytes containing glycine. Electrochim. Acta 56(3), 1399-1408 (2011)

24. J. Bregado-Gutiérrez, A.J. Saldívar-García, H.F. Lopez, Synthesis of Co-50Ni nanocrystals obtained by a modified polyol method. Mater. Lett. 62(6-7), 939-942 (2008)

25. S. Oroujizad, M. Almasi-Kashi, S. Alikhanzadeh-Arani, Sn addition effect on magnetic reversibility of $\mathrm{Co}-\mathrm{Ni}$ alloy nanoparticles based on the FORC results. Mater. Chem Phys 243, 122575 (2020)

26. L. Goth, A simple method for determination of serum catalase activity and revision of reference range. Clin. Chim. Acta 196(23), 143-151 (1991)

27. Y. Sun, L.W. Oberley, Y. Li, A simple method for clinical assay of superoxide dismutase. Clin. Chem. 34(3), 497-500 (1988)

28. G.L. Ellman, Tissue sulfhydryl groups. Arch. Biochem. Biophys. 82(1), 70-77 (1959)

29. D. Jollow, J. Mitchell, Zampaglione Na, Gillette J: Bromobenzeneinduced liver necrosis. Protective role of glutathione and evidence for 3, 4-bromobenzene oxide as the hepatotoxic metabolite. Pharmacology 11(3), 151-169 (1974)

30. H. Ohkawa, N. Ohishi, K. Yagi, Assay for lipid peroxides in animal tissues by thiobarbituric acid reaction. Anal. Biochem. 95(2), 351-358 (1979)

31. M. Almasi-Kashi, S. Alikhanzadeh-Arani, M. Karamzadeh-Jahromi, The role of $\mathrm{Sn}, \mathrm{Zn}$, and $\mathrm{Cu}$ additions on the microwave absorption properties of Co-Ni alloy nanoparticles. Mater. Res. Bull. 118, 110491 (2019)

32. S.S. Kelkar, T.M. Reineke, Theranostics: combining imaging and therapy. Bioconjug. Chem. 22(10), 1879-1903 (2011)

33. D. Ho, X. Sun, S. Sun, Monodisperse magnetic nanoparticles for theranostic applications. Acc. Chem. Res. 44(10), 875-882 (2011)

34. D. Prokopiou et al., Synthesis and characterization of modified magnetic nanoparticles as theranostic agents: in vitro safety assessment in healthy cells. Toxicol. in Vitro 72, 105094 (2021)

35. K. Greish, Enhanced permeability and retention (EPR) effect for anticancer nanomedicine drug targeting, in Cancer nanotechnology. (Springer, 2010), pp. 25-37

36. K.B. Sutradhar, M. Amin, Nanotechnology in cancer drug delivery and selective targeting. Int. Schol. Res. Notices, 2014. 2014.

37. A.R.O. Rodrigues et al., Magnetic liposomes based on nickel ferrite nanoparticles for biomedical applications. Phys. Chem. Chem. Phys. 17(27), 18011-18021 (2015)

38. J. Liu et al., Passive tumor targeting of renal-clearable luminescent gold nanoparticles: long tumor retention and fast normal tissue clearance. J. Am. Chem. Soc. 135(13), 4978-4981 (2013)

39. S. Chatterjee, S. Sarkar, S. Bhattacharya, Toxic metals and autophagy. Chem. Res. Toxicol. 27(11), 1887-1900 (2014)

40. Paunovic, J., et al., Effects of metallic nanoparticles on physiological liver functions. Rev. Adv. Mater. Sci., 2017. 49(2).

41. D. Wang et al., Where does the toxicity of metal oxide nanoparticles come from: the nanoparticles, the ions, or a combination of both? J. Hazard. Mater. 308, 328-334 (2016)

42. R.R. Magaye et al., Acute toxicity of nickel nanoparticles in rats after intravenous injection. Int. J. Nanomed. 9, 1393 (2014)

43. R. Gornati et al., Zerovalent $\mathrm{Fe}, \mathrm{Co}$ and Ni nanoparticle toxicity evaluated on SKOV-3 and U87 cell lines. J. Appl. Toxicol. 36(3), 385-393 (2016)
44. E. Ibrahim et al., Highly biocompatible superparamagnetic Ni nanoparticles dispersed in submicron-sized $\mathrm{C}$ spheres. Carbon $\mathbf{6 3}$, 358-366 (2013)

45. X. Pang et al., Osteopontin as a multifaceted driver of bone metastasis and drug resistance. Pharmacol. Res. 144, 235-244 (2019)

46. Q. Zou et al., Gene2vec: gene subsequence embedding for prediction of mammalian N6-methyladenosine sites from mRNA. RNA 25(2), 205-218 (2019)

47. M. Niu, Y. Lin, Q. Zou, sgRNACNN: identifying sgRNA on-target activity in four crops using ensembles of convolutional neural networks. Plant Mol. Biol. 105(4), 483-495 (2021)

48. D. Guo et al., Synergistic effect of functionalized nickel nanoparticles and quercetin on inhibition of the SMMC-7721 cells proliferation. Nanoscale Res. Lett. 4(12), 1395-1402 (2009)

49. D. Guo et al., Study on the enhanced cellular uptake effect of daunorubicin on leukemia cells mediated via functionalized nickel nanoparticles. Biomed. Mater. 4(2), 025013 (2009)

50. D.-Y. Zhang et al., Cobalt carbide-based theranostic agents for in vivo multimodal imaging guided photothermal therapy. Nanoscale 12(13), 7174-7179 (2020)

51. M. Ahamed et al., Comparative cytotoxic response of nickel ferrite nanoparticles in human liver HepG2 and breast MFC-7 cancer cells. Chemosphere 135, 278-288 (2015)

52. R. Magaye et al., Metallic nickel nanoparticles may exhibit higher carcinogenic potential than fine particles in JB6 cells. PLoS ONE 9(4), e92418 (2014)

53. Z. Lei et al., A full-spectrum-absorption from nickel sulphide nanoparticles for efficient NIR-II window photothermal therapy. Nanoscale 11(42), 20161-20170 (2019)

54. S. Ansari et al., Cobalt nanoparticles for biomedical applications: Facile synthesis, physiochemical characterization, cytotoxicity behavior and biocompatibility. Appl. Surf. Sci. 414, 171-187 (2017)

55. S.M. Peymani-Motlagh et al., Assessing the magnetic, cytotoxic and photocatalytic influence of incorporating $\mathrm{Yb} 3+$ or $\operatorname{Pr} 3+$ ions in cobalt-nickel ferrite. J. Mater. Sci. Mater. Electron. 30(7), 6902-6909 (2019)

56. F. Zheng et al., Comparison of the neurotoxicity associated with cobalt nanoparticles and cobalt chloride in Wistar rats. Toxicol. Appl. Pharmacol. 369, 90-99 (2019)

57. L. Kong et al., Nickel nanoparticles exposure and reproductive toxicity in healthy adult rats. Int. J. Mol. Sci. 15(11), 21253 21269 (2014)

58. D. Zhang et al., Detecting image seam carving with low scaling ratio using multi-scale spatial and spectral entropies. J. Vis. Commun. Image Represent. 48, 281-291 (2017)

59. J. Wang et al., Big data service architecture: a survey. J. Internet Technol. 21(2), 393-405 (2020)

60. F. Yu et al., A robust and fixed-time zeroing neural dynamics for computing time-variant nonlinear equation using a novel nonlinear activation function. Neurocomputing 350, 108-116 (2019)

61. M. Duan et al., A novel multi-task tensor correlation neural network for facial attribute prediction. ACM Trans. Intell. Syst. Technol. (TIST) 12(1), 1-22 (2020)

Publisher's Note Springer Nature remains neutral with regard to jurisdictional claims in published maps and institutional affiliations. 


\section{Authors and Affiliations}

\section{Saman Sargazi ${ }^{1}$ - Mohammad Reza Hajinezhad ${ }^{2}$. Abbas Rahdar ${ }^{3}$. Mahwash Mukhtar ${ }^{4}$. Milad Karamzadeh-Jahromi ${ }^{5}$. Mohammad Almasi-Kashi, ${ }^{5,6}$ Sima Alikhanzadeh-Arani ${ }^{6,7}$. Mahmood Barani ${ }^{8}$. Francesco Baino 9 (D)}

Saman Sargazi

sgz.biomed@gmail.com

Mohammad Reza Hajinezhad

hajinezhad@uoz.ac.ir

Mahwash Mukhtar

mukhtar.mahwash@pharm.u-szeged.hu

Milad Karamzadeh-Jahromi

milad71karamzade@yahoo.com

Mohammad Almasi-Kashi

almac@kashanu.ac.ir

Sima Alikhanzadeh-Arani

s.alikhanzadeh@cfu.ac.ir

Mahmood Barani

mahmoodbarani7@gmail.com

1 Cellular and Molecular Research Center, Research Institute of Cellular and Molecular Sciences in Infectious Diseases, Zahedan University of Medical Sciences, 98167-43463 Zahedan, Iran
2 Basic Veterinary Science Department, Veterinary Faculty, University of Zabol, P. O. Box. 98613-35856, Zabol, Iran

3 Department of Physics, University of Zabol, P. O. Box. 98613-35856, Zabol, Iran

4 Faculty of Pharmacy, Institute of Pharmaceutical Technology and Regulatory Affairs, University of Szeged, Szeged 6720, Hungary

5 Department of Physics, University of Kashan, 87317-51167 Kashan, Iran

6 Institute of Nanoscience and Nanotechnology, University of Kashan, 87317-51167 Kashan, Iran

7 Farhangian University, Tehran, Iran

8 Medical Mycology and Bacteriology Research Center, Kerman University of Medical Sciences, 76169-13555 Kerman, Iran

9 Institute of Materials Physics and Engineering, Department of Applied Science and Technology, Politecnico di Torino, Turin, Italy 